\title{
Development process of micropores partial discharge of silicon rubber in prefabricated cable joint
}

\author{
Wenzhi Chang*, Fei Du*, Jiangang Bi*, Jin Shao**, \\ Jiang Peng ${ }^{* *}$, Jiping Liu ${ }^{* *}$, Haoming Wang ${ }^{* *}$
}

\begin{abstract}
To clarify the relationship between microporous defects and the development of partial discharge(PD) in silicone rubber, meanwhile evaluate insulation state of the prefabricated power cable joints quantitatively, the microporous defect is made based on real power cable joint, and PD signals are measured by step test method with the development of the defect. The discharge number, total energy and average energy of PD is gained to calculate the curves changed over time, based on which the breakdown process is divided into 4 stages, then phased spectra and grayscale maps are calculated for reflection of PD repetition rate and average energy in phase domain. Phase width, skewness of spectrum and coefficient of variation of parameter are employed to indicate the spectra statistical shape feature in each stage. The study indicates that the shape features of spectra are changed during the development of defect, the 3 features parameters are monotonously changed, and the changing rate is significantly higher at the end of the breakdown process. Evaluation method for micropores PD of silicon rubber is proposed based on the analysis of the parameters and the trend of PDs development.
\end{abstract}

K e y w or d s: cable joint, silicon rubber, partial discharge, micropore defect, state monitoring

\section{Introduction}

Because of the random factors due to manufacturing defects, transportation or installation link, micropores or cracks exist in the silicon rubber of the prefabricated power cable joints which finally lead to breakdown under long-term applied voltage loads. Partial discharge (PD) occurs during the insulation deterioration and can be measured, therefore, the insulation state can be evaluated by PD based on the relationship between microporous defects development and the corresponding PD signals.

In the industry, the development patterns of defects in silicon rubber are widely studied. In [1-5], the tree inception voltage and the shape of trees caused by pinpoints with different radius of curvatures are studied, and the aging characteristics of silicon rubber and trees are studied as well, which indicate that the initial tree is formed with the sign of single channel, then is formed with 4 kinds of shapes. In $[5,6]$, based on the defects of bubble and air gap, the initial characteristics of trees under line frequency voltage is studied. In [7-12], polymethyl methacrylate (PMMA) is used to simulate acicular space, and the relationship between the length of trees and corresponding PD signals are quantificationally studied, which indicates that PD and trees are closely connected. In $[7,8]$, the aging model of power cables are studied, which indicates that the characteristics of aging, the length of trees and PD satisfy function relation. In [13], the PD characteristics are studied based on statistical methods, the development of trees is connected to insulator aging under different applied voltage. In [14], based on the PD characteristic in PE insulator, the unreliability of insulation diagnosis with the maximum discharge is confirmed.
In summary, the previous studies focus on the mechanisms of micropore or trees in silicon rubber, and the analysis on the procedure of trees and PD phenomenon. The association characteristics between the trees process and PD in each development stage are not widely studied, and the experimental and theoretical basics are inadequate for cable joint insulation state evaluation based on PD.

In this paper, the real microporous defect models in $35 \mathrm{kV}$ cable joints are designed, the PD signals of the whole breakdown procedure is measured by coaxial cylinder sensor, then PD development stages are divided and statistical characteristics are calculated to study the process of defects in each stage, and finally the evaluation method is proposed.

\section{Experimental system}

\subsection{Experimental circuit}

The experimental circuit for PD test is shown in Fig. 1(a), and the photo of the platform is shown in Fig. 1(b). The system consists of a $50 \mathrm{kV}$ corona free test transformer, the defect model, PD measurement system $(20 \mathrm{kHz}-200 \mathrm{kHz})$, an $800 \mathrm{pF}$ coupling capacitor and an acquisition card $(250 \mathrm{MS} / \mathrm{s} / \mathrm{ch}$, bandwidth $125 \mathrm{MHz}$, vertical resolution 8 bits).

\subsection{Defect model in cable joint}

Considering with the real cable joint, a needle with diameter $0.1 \mathrm{~mm}$ is stuck into the main insulation, and is pulled out while the tip reaches the semi conductive adhesive tape. As the silicon rubber has well flexibility, the silicon rubber around the crack can gather under

* China Electric Power Research Institute, Beijing 100192 China, dufei@epri.sgcc.com.cn, ** State Grid Corporation of China, Beijing 100031, *** State Grid Tianjin Electric Power Research Institute, Tianjin, 300384 China, dufei@epri.sgcc.com.cn 


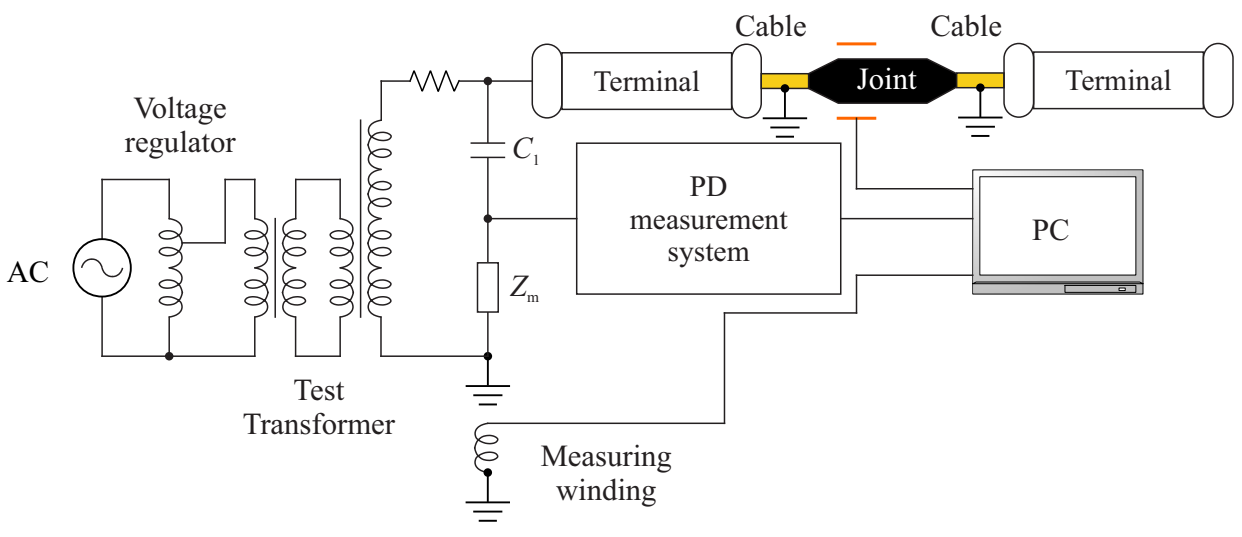

Fig. 1. Measurement setup

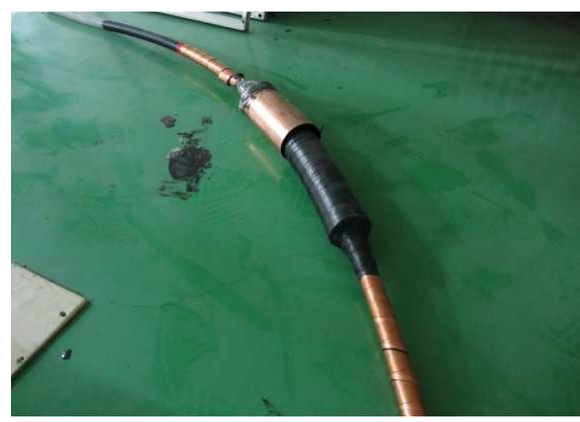

Fig. 3. Coaxial cylinder PD sensor

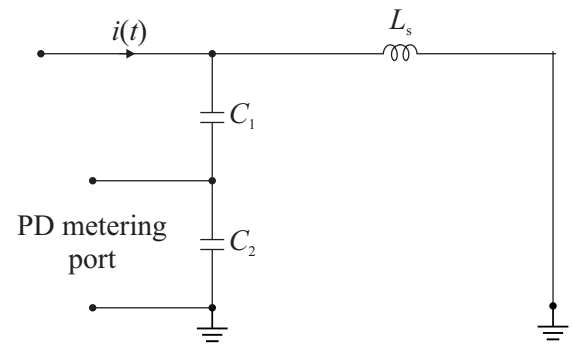

Fig. 4. Circuit measurement model in high frequency

high pressure, and then the micro-pore defect model is installed, as shown in Fig. 2.

\subsection{Coaxial cylinder PD sensor}

The $0.8 \mathrm{~mm}$ thick red copper is welded as a hollow cylindrical shell, whose internal diameter is $20 \mathrm{~mm}$ and whose length is longer than the external diameter of the joint, and the length of the shell is about half of the axial length of the joint. Epoxy resin is used to fix the copper, and finally the coaxial cylinder PD sensor is shown in Fig. 3.

The PD source is assumed as the pulse point source, the pulse current propagates in different directions along the metal shielding layer. As the resistance of metal shielding layer can be ignored, the circuit of the measurement system under high frequency is shown in Fig. 4, in which $L_{\mathrm{s}}$ represents lumped inductor along the shield ground, $C_{1}$ represents space capacitance between the sensor and the shielding layer, $C_{2}$ represents the lumped capacitance of coaxial cable and $i(t)$ represents the pulse $\mathrm{PD}$ current in the metal shielding layer.

\subsection{Experiment progress} nels.

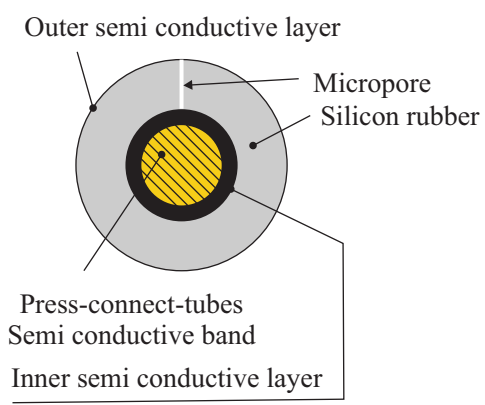

Fig. 2. Diagram of microporous defect

As the inductor $L_{\mathrm{s}}$ works as high resistance in high frequency, the high frequency component of $i(t)$ goes through the series branch consists of $C_{1}$ and $C_{2}$ which works as voltage divider, and PD measurement is achieved by the voltage applied on the $C_{2}$. The upper-cut-off frequency of the PD sensors is $50 \mathrm{MHz}$, and the lower cut-off frequency of the PD sensor is $150 \mathrm{kHz}$.

Firstly, the PD sensor is assembled on the cable joint and the measurement system is calibrated. Secondly, high voltage with power frequency is applied on the defect model. And then the voltage is raised step by step, and stable PD signal is detected while the voltage reaches $14 \mathrm{kV}$, and disappears while the voltage is little declined, therefore, the PD inception voltage is selected as $14 \mathrm{kV}$. The voltage is raised by the step of $2 \mathrm{kV}$, and kept 100 minutes in each step, the insulation breakdown happens while the voltage is $24 \mathrm{kV}$ and is held 90 minutes. Finally, the whole progress costs 9 hours 50 minutes. In the experiment, while the applied step-voltage is high enough, the deterioration of insulator will development, if the deterioration state becomes stable, the time cost of which is recorded. At the end of the experiment, as the insulation is nearly destroyed, the applied voltage is decreased slightly to slow down the development process which helps for observation.

\section{PD patterns caused by microporous defect}

\subsection{Development of PD caused by microporous defect}

To observe the discharge trace, the silicon rubber around the defect is cut into slices, as shown in Fig. 5 . Crackles are formed from the penetrating microporous, and combined with carbonization trace, which namely indicates that the PD happens in the crackle-shaped chan-

To further observe the discharge trace, the slices are cut along the crackles, as shown in Fig. 6. The surface becomes rough with obvious traces of corrosion, and obvious black carbon particles are left. As after the crackles 


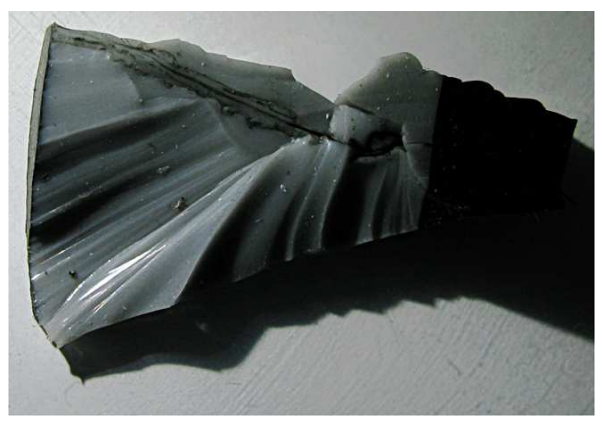

Fig. 5. Photo of disintegrated defect: (a) - corrosion on the surface, (b) - carbon particles precipitation
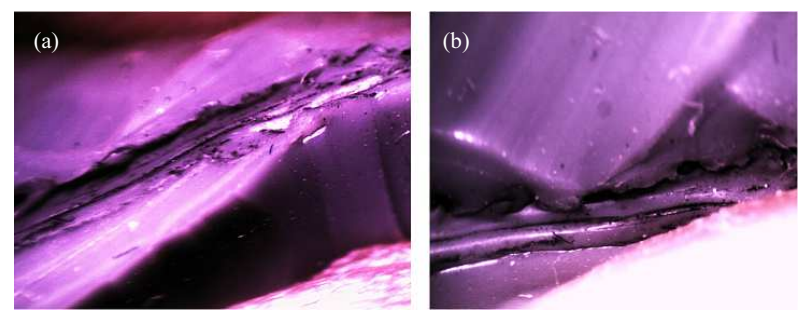

Fig. 6. Photos of interior of the crack by microscope

Table 1. Stages of PD caused by micropores

\begin{tabular}{lccc}
\hline Stage & Time $/ \min$ & Span $/$ min & Applied voltage $/ \mathrm{kV}$ \\
\hline I & $0 \sim 143$ & 143 & $14 / 16$ \\
II & $143 \sim 350$ & 208 & $16 / 18 / 20$ \\
III & $350 \sim 510$ & 161 & $20 / 22 / 24$ \\
IV & $510 \sim 590$ & 81 & $24 / 23$ \\
\hline
\end{tabular}

along the direction of electric field are formed, the uniformity of the medium is destroyed, then air enters the gap which forms a surface between air and silicon rubber. While the applied voltage is higher, electric-field distribution becomes more inhomogeneous, discharge happens in the most inhomogeneous area as well. Silicon rubber is corroded by $\mathrm{O}_{3}$ and nitrogen oxide caused by discharge in air, and the phenomenon is shown in Fig. 6(a). Discharge along the surface further develops as creepage along the direction of electric field, and finally develops as arc discharge when the creepage distance increases to the electrode spacing. The energy in the arc channel releases huge heat and breaks the molecular chain, which forms ablative marks and carbon elements are separated out, as shown in Fig. 6(b).

To study the quantitative relationship between microscope defect and PD development, the discharge number $N$ in unit time $t$, total energy $W_{\text {total }}$, and average energy $W_{\text {ave }}$ are concerned firstly, as

$$
\begin{aligned}
W_{\text {total }} & =\sum_{i=1}^{N} W_{i}, \\
W_{\text {ave }} & =W_{\text {total }} / N
\end{aligned}
$$

where, $W_{i}$ represents the energy during the $i^{\text {th }}$ discharge in unit time $t$. It is assumed that the apparent charge in the $i^{\text {th }}$ discharge during $t$ is $q_{i}$, and the initial voltage is $u_{i}$, the energy is

$$
W_{i}=\frac{1}{2} q_{i} u_{i}
$$

The unit time is considered as 5 minutes, then the relationship between $N-t, W_{\text {ave }}-t$ and $W_{\text {total }}-t$ is shown in Fig. 7.
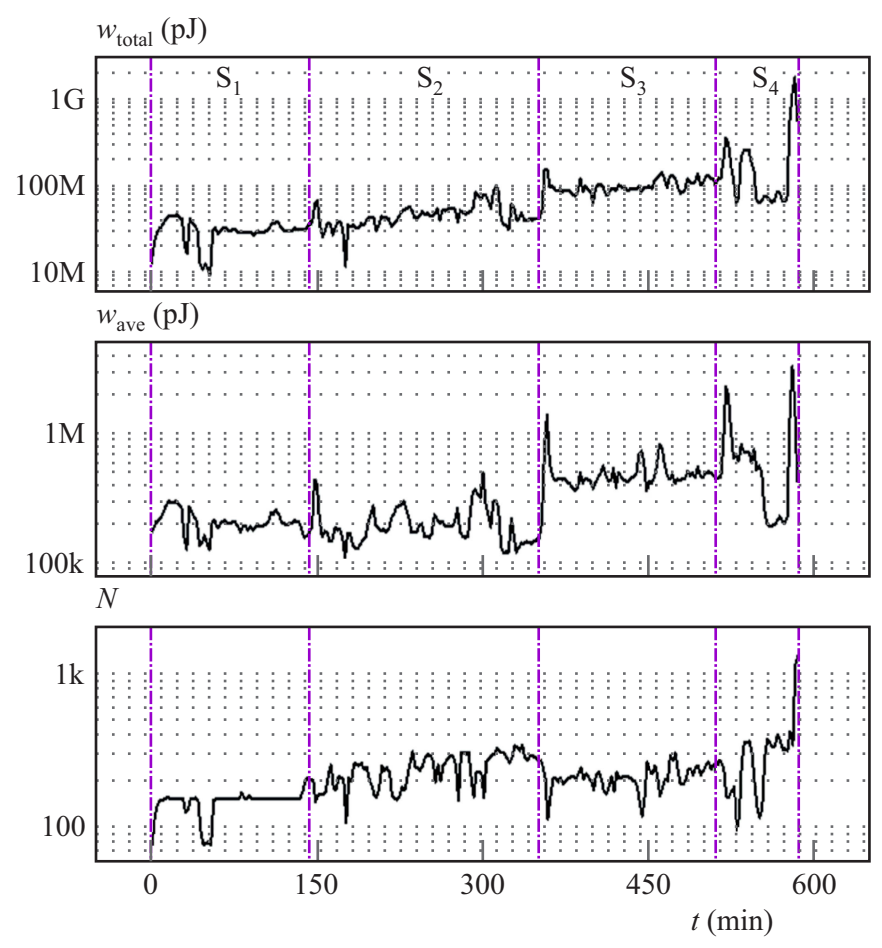

Fig. 7. Curves of $\mathrm{PD}$ characteristics in time domain

Figure 7 indicates that $N, W_{\text {ave }}$ and $W_{\text {total }}$ change obviously in time domain at the beginning of discharge, but then keep stable respectively. Then the amplitudes of 3 characteristics becomes slightly larger. Obvious turning points can be confirmed after a period and then keep stable again. Finally, the curves changed heavily, and raise obviously. According to the change patterns of the curves, the PD development are divided into 4 stages, as shown in Fig. 7, the applied voltage in each stage is shown in Tab. 1.

\subsection{Statistical characteristics in each stage}

According to section 3.1, combined with statistical analysis in phase domain, the relationship between the development of micropore defect and PD can be built, namely the distribute regulation of $\mathrm{PD}$ repetition rate $n$ and average discharge magnitude $q_{\text {ave }}$ in $\varphi$ domain, or the two-dimensional spectrum of $\varphi-n$ and gray scale map of $\varphi-q_{\text {ave }}$. On the other hand, the phase of the AC voltage $\left(0^{\circ}-360^{\circ}\right)$ is equally divided into 128 sections, $n$ and $q_{\text {ave }}$ of each section are counted respectively, namely to achieve describing PD detail features and the features in different stages based on extreme normalization and maximum normalization maps. In addition, phase width can be calculated by the sum of phase length when $q_{\text {ave }}$ is not zero according to $\varphi-q_{\text {ave }}$ spectrum. 

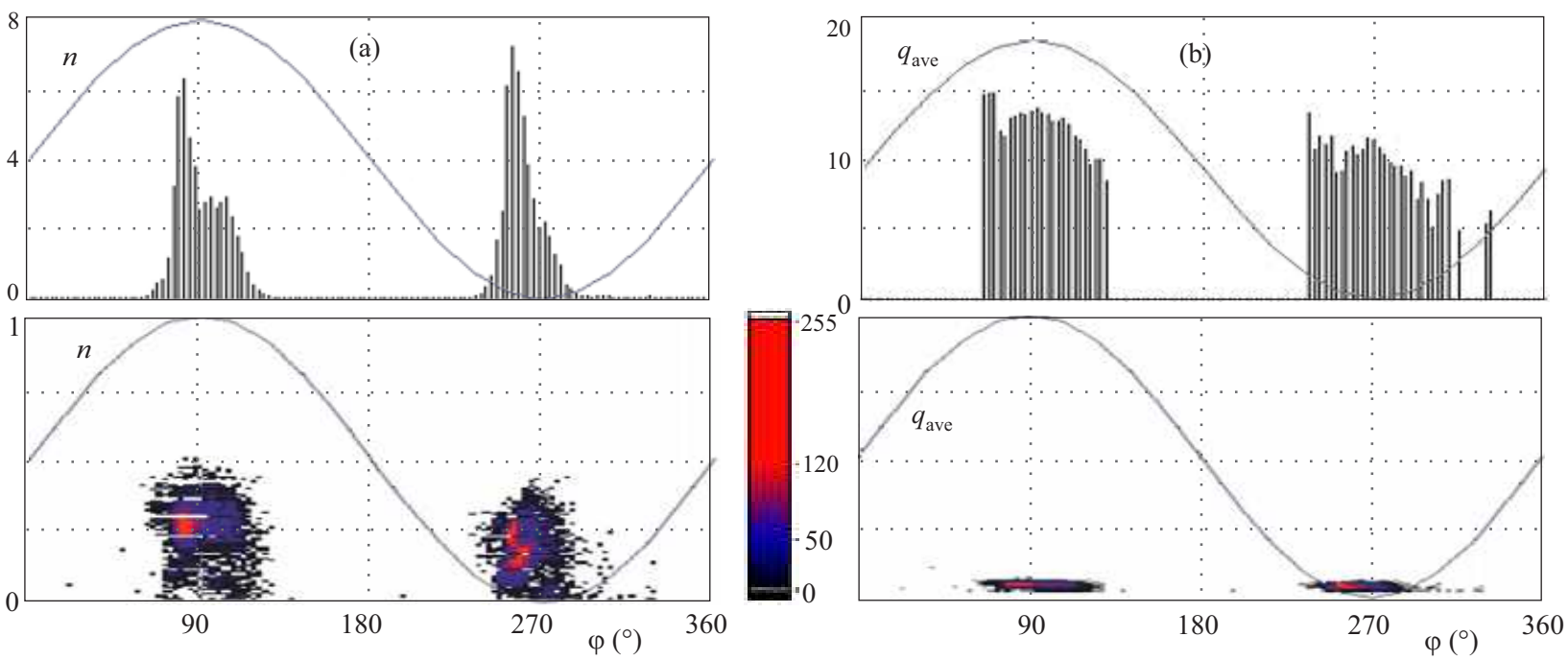

Fig. 8. Phase characteristics spectra in stage I: (a) - , (b) - , (c) - with extreme normalization, (d) - with maximum normalization
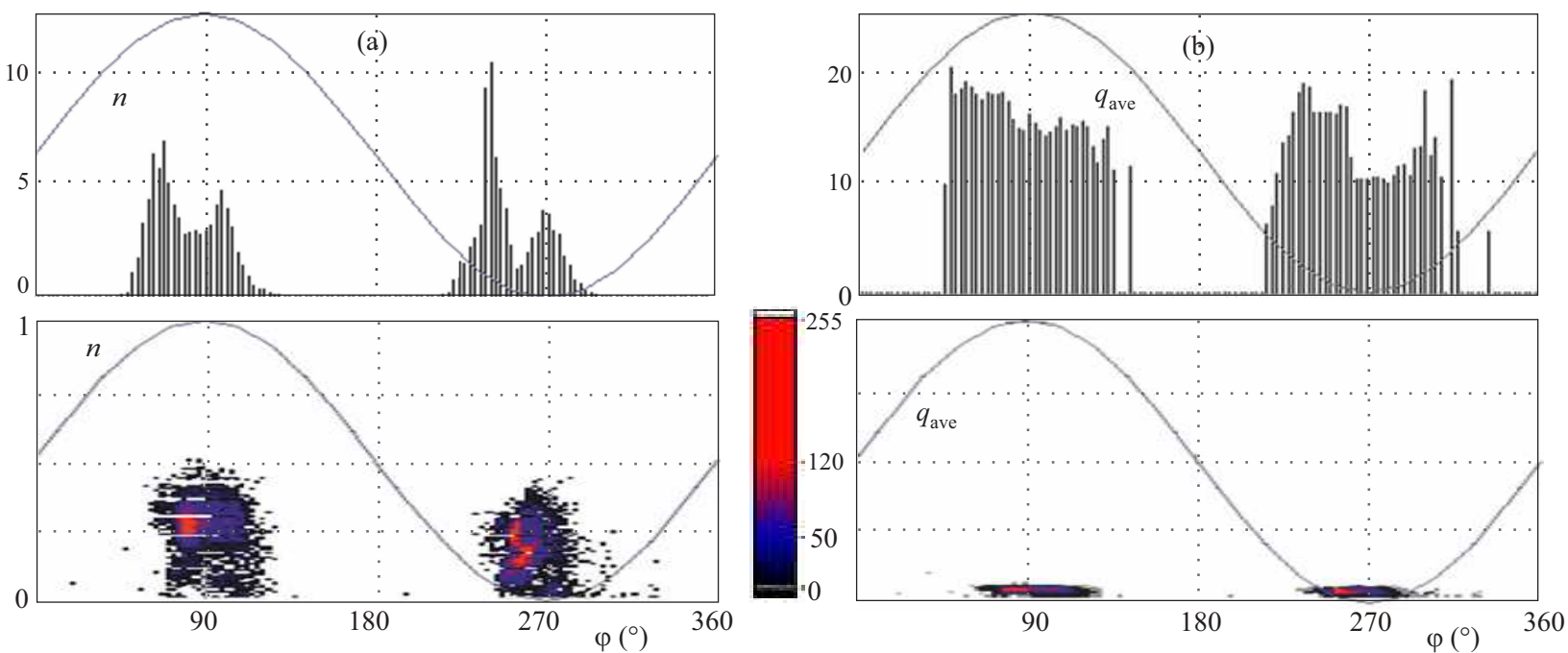

Fig. 9. Phase characteristics spectra in stage II

\subsubsection{Stage I}

The stage I lasts 143 minutes with the applied voltage $14 \mathrm{kV}$ and $16 \mathrm{kV}$, the $\varphi-n, \varphi-q_{\text {ave }}$ spectra and gray scale map is shown in Fig. 8.

Considering all average energy $q_{\text {ave }}$ which is represented by $V$, the $i^{\text {th }}$ value of $V$ is represented by $V(i)$, the maximum value of $V$ is $\max (V)$ and the minimum value of $V$ is $\min (V)$, the maximum normalized value of $V(i)$ is represented by $V_{n}(i)$.

$$
V_{n}(i)=\frac{V(i)-\min (V)}{\max (V)-\min (V)} .
$$

The extreme normalized value of $V(i)$ is represented by $V^{*}(i)$

$$
\begin{aligned}
V^{\prime}(i) & =\frac{V(i)-\bar{V}}{\sqrt{\frac{1}{n} \sum_{i=1}^{n}(V(i)-\bar{V})^{2}}}, \\
V^{*}(i) & =\frac{V^{\prime}(i)-\min \left(V^{\prime}(i)\right)}{\max \left(V^{\prime}(i)\right)-\min \left(V^{\prime}(i)\right)} .
\end{aligned}
$$

Figure 8(a) and (b) indicate that the PDs mainly happen in $62^{\circ} \sim 127^{\circ}$ during the positive half cycle and happen in $234^{\circ} \sim 30^{\circ}$ during the negative half cycle whose repetition rate is lower. There is single peak in each half-cycle in $\varphi-n$ spectrum and both $\mathrm{n}$ and $q_{\text {ave }}$ become the largest at the maximum voltage. The extreme normalization gray-scale map indicates that PDs happen around the $90^{\circ}$ and $270^{\circ}$ in phase domain and energy distributes uniformly. The maximum normalization map fails to distinguish features as the amplitudes are too low.

\section{$3.2 .2 \mathrm{Stage} \mathrm{II}$}

The stage II lasts 208 minutes with the applied voltage of $16 \mathrm{kV}, 18 \mathrm{kV}$ and $20 \mathrm{kV}$. The spectra are shown in Fig. 9.

Figure $9(\mathrm{a})$ and (b) indicate that PDs mainly happen in $42^{\circ} \sim 132^{\circ}$ and $214^{\circ} \sim 315^{\circ}$ during each half cycle, (5) and the width in phase domain is larger than which in stage I. There are double peaks in each half cycle of $\varphi-n$ spectrum. The extreme normalization gray-scale map in- 

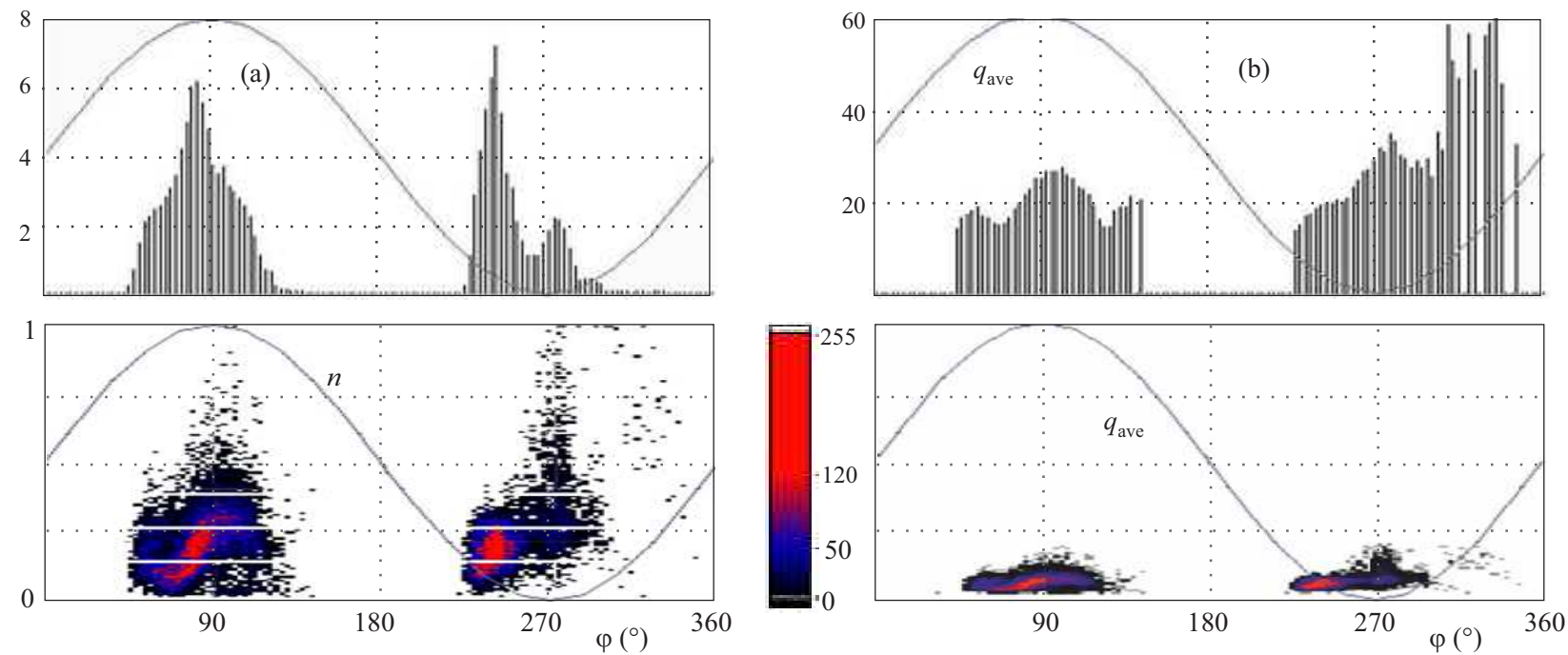

Fig. 10. Phase characteristics spectra in stage III
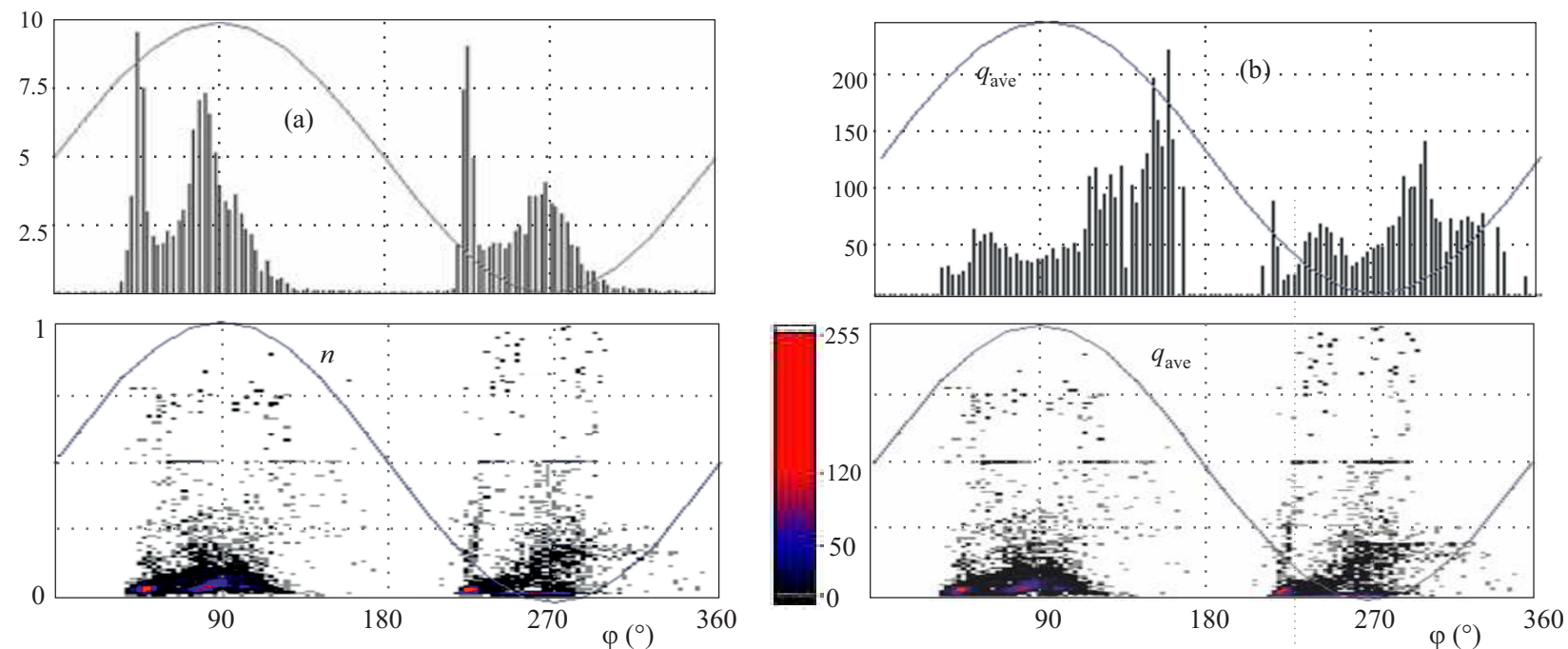

Fig. 11. Phase characteristics spectra in stage IV

Table 2. Distribution in phase interval of each stage

\begin{tabular}{lcccc}
\hline & \multicolumn{2}{c}{ Phase section $\left(^{\circ}\right)$} & \multicolumn{2}{c}{ Phase width $\left(^{\circ}\right)$} \\
\cline { 2 - 5 } Stage & $\begin{array}{c}\text { Positive } \\
\text { cycle }\end{array}$ & $\begin{array}{c}\text { Negative } \\
\text { cycle }\end{array}$ & $\begin{array}{c}\text { Positive } \\
\text { cycle }\end{array}$ & $\begin{array}{c}\text { Negative } \\
\text { cycle }\end{array}$ \\
\hline I & $62 \sim 127$ & $234 \sim 307$ & 66 & 74 \\
II & $42 \sim 132$ & $214 \sim 315$ & 91 & 102 \\
III & $45 \sim 144$ & $225 \sim 335$ & 100 & 120 \\
IV & $36 \sim 166$ & $208 \sim 338$ & 131 & 131 \\
\hline
\end{tabular}

dicates that the energy is lower but denser when the applied voltage is rising and is similar with what in the negative half cycle. Although the amplitudes of maximum normalization are larger, it still fails to clarify the PD features.

\subsection{3 $\mathrm{S}$ t a g e II I}

The stage III lasts 161 minutes with the applied voltage of $20 \mathrm{kV}, 22 \mathrm{kV}$ and $24 \mathrm{kV}$. The spectra are shown in Fig. 10.
Figure 10 indicates that PDs mainly happen in $45^{\circ} \sim$ $144^{\circ}$ and $225^{\circ} \sim 335^{\circ}$ during each half cycle, and the width is nearly as same as which in section II. There is a single bell-shaped peak in positive half cycle in $\varphi-n$ spectrum and 2 peaks in the negative half cycle. In $\varphi-q_{\text {ave }}$ spectrum, the envelope is wavy in positive half cycle and is ramp in negative half cycle. The extreme normalization map indicates that the lower PDs are densely distributed in positive half cycle and voltage reverse increasing area. PD energy in both gray scale maps becomes larger and distributes sparsity.

\subsection{4 $\mathrm{Stage}$ I V}

The stage IV lasts 77 minutes with the applied voltage of $24 \mathrm{kV}$ and $23 \mathrm{kV}$. The spectra are shown in Fig. 11 .

Figure 11(a) and (b) indicate that PDs mainly happen in $36^{\circ} \sim 166^{\circ}$ and $208^{\circ} \sim 338^{\circ}$ during each half cycle, whose width is much longer than the other 3 stages, and the distribution patterns are close to zero crossing point. In $\varphi-n$ spectrum, there are 2 peaks in positive half cycle, the first peak is sharper and higher, there are 


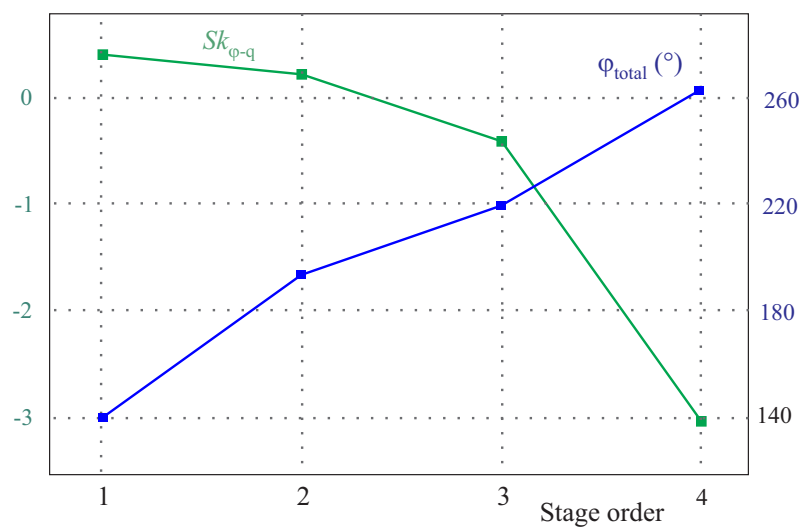

Fig. 12. Phase width and skewness of $\varphi-\mathrm{q}_{\text {ave }}$ spectra in negative half cycle in each stage

2 peaks in negative half cycle as well, but the difference in amplitude is greater. In $\varphi-q_{\text {ave }}$ spectrum, the envelope of each half cycle increases undulately. The features of both extreme and maximum normalization gray scale maps are the same, the envelope in positive half cycle is triangular and lower, and the envelope of higher energy forms sparse spectrum. The gray scale maps in stage IV are more sparse with larger amplitude.

\section{Statistical characteristics and correlation between microporous defect and PD}

\subsection{Phase width of PD}

Based on the PD spectra in each stage, the phase width in which $q_{\text {ave }}>10 \mathrm{pC}$ is calculated as shown in Table 2 . With the development of $\mathrm{PD}$, the phase section gets closer to $0^{\circ}$ in positive half cycle, and to $180^{\circ}$ in negative half cycle.

The total phase width in each stage $\varphi_{\text {total }}$ is calculated according to Table 2, as shown in Fig. 12.

\subsection{Skewness of $\varphi-q_{\text {ave }}$ spectra}

The skewness of $\varphi-q_{\text {ave }}$ spectra, which indicates the deviation of spectra shape compared with normal distribution is

$$
S_{k}=\frac{\sum_{i=1}^{w}\left(x_{i}-\mu\right)^{3} \times p_{i}}{\sigma^{3}}
$$

where $x_{i}$ represents the phase of the $i^{\text {th }}$ window, $p$, $\mu$ and $\sigma$ represent the probability, average value and standard deviation respectively.

$$
p_{i}=\frac{y_{i}}{\sum_{i=1}^{w} y_{i}}, \mu=\sum_{i=1}^{w} p_{i} \varphi_{i}, \quad \sigma=\sqrt{\sum_{i=1}^{w} p_{i}\left(\varphi_{i}-\mu\right)^{2}}
$$

where, $y_{i}$ represents the ordinate of the spectrum, namely the $\mathrm{PD}$ value.

For the PD caused by microporous defect, the skewness $S_{k}$ decreases stable from stage I to stage III, and decreases faster from stage III to stage IV, as shown in

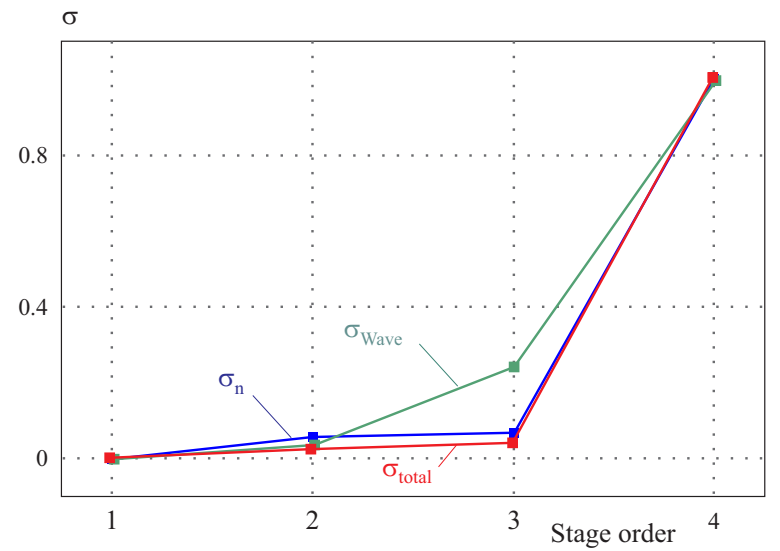

Fig. 13. Coefficient of variations in each stage

Fig. 12. Therefore, the envelope of $\varphi-q_{\text {ave }}$ spectrum deflects towards right side heavily at the end of breakdown procedure.

\subsection{Coefficient of variation based on tendency chart}

The change pattern of parameters such as $N, W_{\text {ave }}$ and $W_{\text {total }}$ are obviously different in each stage. To quantificationally analyze the difference of the same parameters in different stages, coefficient of variation based on tendency chart is employed as coefficient of variation describing the ratio of the maximum value and minimum value of a certain function

$$
\delta_{i}=\frac{f_{\max }\left(\varphi_{i}\right)}{f_{\min }\left(\varphi_{i}\right)}
$$

where $i=1,2,3,4$ represents the number of the stages, $f\left(\varphi_{i}\right)$ represents the function based on the curves of $N$ , $W_{\text {ave }}$ or $W_{\text {total }}$ of the $i^{\text {th }}$ stage. $\varphi_{i}$ represents the phase value whose range belongs to the $i^{\text {th }}$ stage. The coefficients of variations are shown in Fig. 13.

Figure 13 indicates that the coefficients of variations increase with the development of PD, and increase suddenly at the end of stage III.

\section{Segmental evaluation method for the severity of PD}

According to sections 3 and 4 , phase width, $S_{k}$ (skewness of $\varphi-q_{\text {ave }}$ spectra), and $\delta$ (the coefficient of variation of $N, W_{\text {ave }}$ and $\left.W_{\text {total }}\right)$ are monotonous changing with the development of PD caused by micropores defect. The growth rate of phase width is stable during the whole breakdown procedure. $S_{k}$ is decreasing with low speed from stage I to stage III, and suddenly faster in stage IV, conversely, $\delta$ increases with low speed in the first three stages, and suddenly enlarge at last stage. Based on the changing patterns of the statistical parameters, the PD severity caused by micropores defect can be divided into 3 degrees. 
(1) Initial stage of PD. The initial stage of PD corresponds to the stage I in section 3 , during which crackles are formed by micropore along direction of electric field, and the surface of air and silicon rubber leads to uneven electric-field distribution, then PD occurs at the most uneven area. The statistical characteristics satisfy the analysis in section 3.2.1, namely $N$, $W_{\text {ave }}$ and $W_{\text {total }}$ fluctuate obviously at the beginning of PD but rapidly keep stable. PD monitor should be stepped to further master the development and change of PD.

(2) Developing stage of PD. The developing stage of PD corresponds to the stage II and stage III, the changes of parameters match the analysis in section 3.2 .2 and section 3.2.3. Phase width and $\delta$ increase slowly, but $S_{k}$ decreases. $N, W_{\text {ave }}$ and $W_{\text {total }}$ are substantially retained. The creeping voltage occurs along a larger surface crack. Reasonable maintenance strategy in advance should be made based on the PD monitor result.

(3) Severe deterioration stage of PD. The severe deterioration stage of $\mathrm{PD}$ corresponds to the stage $\mathrm{IV}$, and the changes of parameters match the analysis in section 3.2.4. During this stage, phase width is enlarged, the increasing speed of $\delta$ and the decreasing speed of $S_{k}$ are obviously faster respectively, and inflection points exist in the curves of $\delta$ and $S_{k}$. There are drastic changes in the amplitude of $N, W_{\text {ave }}$ and $W_{\text {total }}$ with the characteristics of high and low oscillations. The discharge area increases evidently, the creeping distance along the interface between the crack and the silicone rubber is close to the length between the cable core and the shield, the breakdown will occur and the cable joint should be changed as soon as possible.

\section{Conclusions}

(1) To further study the breakdown accident caused by micropores or cracks in the cable joint, the microporous defect model in $35 \mathrm{kV}$ cable joint is made. PD signals are gained and analyzed in PD experiments based on step test method, which informs the characteristics and phenomenon in different PD development stages.

(2) The development of PD is divided into 4 stages. Based on the spectra of each stage, the phase width, spectra of $\varphi-q_{\text {ave }}$, skewness of $\varphi-q_{\text {ave }}$ spectra, and coefficient of variation based on tendency chart are calculated to describe the features and characteristics.

(3) The studies indicate that the curves of phase width remain stable during the breakdown procedure. $S_{k}$ keeps decreasing and decreases especially faster in stage IV. The curves of $\delta$ enlarge slowly in the first three stages, then increase suddenly in the last stage. Based on the change rules, the segmental evaluation method for the severity of PD is finally proposed.

\section{Acknowledgement}

This work was supported by "Research on detection and protection of latent danger of power cable and qual- ity evaluation technology of product construction", the Science and Technology Foundation of State Grid Corporation of China (GY71-18-040).

\section{REFERENCES}

[1] G. Katsuta, A.Toya, K. Muraoka et al, "Development of a Method Partial Discharge Detection in Extra-High Voltage Cross-Linked Polyethylene Insulated Cable Lines", IEEE Transactions on Power Delivery, 1992, vol. 7, no. 3, pp. 1068-1079.

[2] R. Liao, T. Zhou and L. Liu et al, "Experimental Research on Electrical Treeing and Partial Discharge Characteristics of Cross-linked Polyethylene Power Cables", Proceedings of the CSEE, 2011, vol. 31 no. 28, pp. 136-143.

[3] X. Chen, Y. Xu, M. Wang et al, "Propagation and Partial Discharge Characteristics of Electrical Trees in $110 \mathrm{kV}$ XLPE Cable Insulation at High Temperature", High Voltage Engineering, 2012, vol. 38, no. 3, pp. 645-654.

[4] T. Heizmann, T. Aschwanden, H. Hahn et al, "On-Site Partial Discharge Measurements on Premolded Cross-Bonding Joints of 170 kV XLPE and EPR cables", Power Delivery, 1998, vol. 13, no. 2, pp. 330-335.

[5] K. Fukunaga, M. Tan and H. Takehana, "New Partial Discharge Detection Method for Live UHV/EHV Cable Joints", IEEE Transactions on Electrical Insulation, 1992, vol. 27, no. 3, pp. 669-674.

[6] X. Zheng and G. Chen, "Propagation Mechanism of Electrical Tree in XLPE Cable Insulation by Investigating a Double Electrical Tree Structure", Dielectrics \& Electrical Insulation IEEE Transactions, 2008, vol. 15, no. 3, pp. 800-807.

[7] C. Montanari, "Aging and Life Models for Insulation Systems Based on PD Deteetion", IEEE Transactions on Dielectrics and Electrical Insulation, 1995, vol. 2, no. 4, pp. 667-675.

[8] R. Bozzo, G. Gemme and F. Guastavino, "Aging Diagnosis of Insuation Systems by PD Measurements Extraction of Partial Discharge Features in Electrieal Treeing", IEEE Transactions on Eleetrical Insulation, 1998, vol. 5, no. 1, pp. 118-124.

[9] A. Contin, E. Gulski, M. Cacciari et al, "Inference of PD in Electrical Insuation by Charge-Height Probability Distribution-Diagnosis of Insulation System Degradation", IEEE Transactions on Dielectrics and Electrical Insulation, 1998, vol. 5, no. 1, pp. 110-117.

[10] A. Nezar and N. H. Ahmed, "On-Line Versus Off-Line Partial Diseharge Testing in Power Cables", Transmission and Distribution Conference and Exposition, Atlanta, Georgia, IEEE, 2001, pp. 865-870.

[11] W. Chang, C. Yan, C. Li et al, "Assessment of Creeping Discharge Initiated by Metal Particles on the Silicone Rubber/XLPE Interface in Cable Joints", Proceedings of the CSEE, 2015, vol. 30, no. 24, pp. 245-254.

[12] Y. Kamiya, Y. Muramoto and N. Shimizu, "Influence of Absorbed Gas in Silicone Rubber on Electrical Tree Initiation", IEEE International Conference on Solid Dielectrics, IEEE, 2007.

[13] X. R. Chen, X. U. Yang, X. U. Jie et al, "Propagation Stage Characteristics of Electrical Tree in $110 \mathrm{kV}$ XLPE Cable Insulation at $50 \mathrm{~Hz}$ Power Frequency Voltage", Advanced Technology of Electrical Engineering \& Energy, 2010, vol. 80, no. 3, pp. 39-43.

14] S. Tokunaga, T. Tsurusaki, Y. Z. Arief et al, "Partial Discharge Characteristics Till Breakdown for XLPE Cable Joint with an Artificial Defect", Properties \& Applications of Dielectric Materials, Proceedings of International Conf. 2003, pp. 1206-1209.

Received 5 January 2018 